\title{
Cardiac testing in an asymptomatic 42-year-old man
}

Choosing
Wisely
Canada

\section{R. Sacha Bhatia MD MBA, Ciara Pendrith MSc BSc(H), Heather Ross MD MHSc; and the Canadian Cardiovascular Society Choosing Wisely Canada Committee*}

\begin{abstract}
A 42-year-old nonsmoking man presented to his family physician to ask about his cardiac risk. Both the patient's father and grandfather had had fatal myocardial infarctions in their 40s. The patient does not have any symptoms; he walks a couple of kilometers daily and is able to walk two flights of stairs without difficulty. He has had no previous cardiac investigations. His family doctor considers what the appropriate cardiac work-up is for this patient.
\end{abstract}

\section{Does this patient have features that might warrant further investigations?}

The decision to screen an asymptomatic patient for coronary artery disease (CAD) depends on the patient's pretest risk. Risk for CAD may be defined by using the Framingham risk score and divided into three categories: low (10-yr risk of $\mathrm{CAD}<10 \%$ ), intermediate (10-yr risk 10\%-20\%) and high $(10-y r$ risk $>20 \%) .{ }^{1}$ Factors included in the score (www.cvdriskchecksecure.com/ FraminghamRiskScore.aspx) are age, sex, total cholesterol level, high-density lipoprotein (HDL) cholesterol level, smoking status, diabetes, systolic blood pressure and treatment for hypertension.

Risk factors may be categorized as modifiable or nonmodifiable. Modifiable risk factors for CAD include tobacco use or smoking history, dyslipidemia, diabetes, hypertension, chronic kidney disease, physical inactivity, poor diet, obesity or metabolic syndrome and depression. Nonmodifiable risk factors include age (men $\geq 40 \mathrm{yr}$; women $\geq 50 \mathrm{yr}$ or postmenopause), ethnicity (i.e., First Nations or South Asian) and family history of premature cardiovascular disease. Physical examination has low sensitivity for the detection of CAD, but a body mass index (BMI) greater than 27, elevated blood pressure, cardiovascular abnormalities (e.g., murmurs, gallops, bruits, absent pulses) or evidence of clinical heart failure (i.e., elevated jugular venous pressure) might alter the probability of underlying disease. ${ }^{2}$ These risk factors, particularly those not part of the Framingham risk score, and findings on physical examination may suggest the presence of $\mathrm{CAD}$, and further investigations may be warranted.

This patient's family history of premature cardiac disease is an unmodifiable risk factor, and further investigations to assess cardiac risk may be needed.

\section{Does this patient require laboratory investigations to screen for risk factors?}

The 2012 Canadian Cardiovascular Society dyslipidemia guideline recommends screening for CAD risk factors in all men more than 40 years of age and in women who are either more than 50 years of age or postmenopausal. ${ }^{3} \mathrm{~Pa}$ tients of First Nations or South Asian descent are at increased risk of CAD, and consideration should be given to screening at an earlier age. ${ }^{3}$ The guideline also recommends screening for cardiac risk factors in adults of any age with the following risk factors: rheumatoid arthritis, systemic lupus erythematosus, psoriatic arthritis, ankylosing spondylitis, inflammatory bowel disease, chronic obstructive pulmonary disease, chronic HIV infection, chronic kidney disease, abdominal aneurysm and erectile dysfunction.

Laboratory investigations should include measurement of fasting blood glucose, low-density lipoprotein (LDL) cholesterol, HDL cholesterol, non-HDL lipids, triglycerides and estimated glomerular filtration rate. ${ }^{3}$ As per the guideline, ${ }^{3}$ this patient (male sex, age $>40 \mathrm{yr}$ ) should undergo screening for these cardiac risk factors.

\section{Should this patient undergo screening with electrocardiography?}

As mentioned previously, the patient's Framingham risk score should be calculated before any diagnostic tests are ordered. In patients with low risk and no symptoms, electrocardiography
Competing interests: None declared.

This article has been peer reviewed.

The authors have obtained patient consent.

*Members of the committee are listed at the end of the article.

Correspondence to: Sacha Bhatia, sacha.r.bhatia @wchospital.ca

CMAJ 2015. DOI:10.1503 /cmaj.141479 
(ECG) has not been found to accurately restratify a patient's risk of CAD; however, some small cohort studies have suggested a higher rate of clinical events with resting abnormalities on ECG. ${ }^{4}$ Because of the low prevalence of cardiac events in a low-risk population, screening with ECG will likely not change a patient's risk stratification to a degree that would prompt a change in management.

On this basis, the US Preventive Services Task Force has concluded that outpatients with low risk and no symptoms should not undergo routine screening for $\mathrm{CAD}$ with $\mathrm{ECG},{ }^{5}$ a position that is supported by Choosing Wisely Canada (Box 1). ${ }^{6}$ Furthermore, insufficient evidence exists to routinely recommend such screening for patients with intermediate or high risk and no symptoms, although screening decisions should be individualized. ${ }^{5}$

\section{Should this patient undergo an exercise test or stress imaging?}

Although there are no randomized controlled trials comparing clinical outcomes of patients with no symptoms who undergo screening by exercise ECG with patients who do not, large cohort studies have assessed the risk of death or myocardial infarction in this patient population, deemed low risk, at less than $1 \%$ per year. $^{7}$ Exercise testing has poor accuracy to diagnose hemodynamically important $\mathrm{CAD}$ in patients with no

\section{Box 1: Choosing Wisely Canada recommendations} on electrocardiography and cardiac imaging ${ }^{6}$

- Don't perform stress imaging or advanced noninvasive imaging in the initial evaluation of patients with no cardiac symptoms unless high-risk markers are present (diabetes in patients aged $>40 \mathrm{yr}$; peripheral arterial disease; $>2 \%$ annual risk for coronary artery disease events).

- Don't perform annual stress imaging or advanced noninvasive imaging as part of routine follow-up in asymptomatic patients.

- Don't order annual electrocardiograms (ECGs) for patients at low risk $(10-y r$ risk $<10 \%)$ with no symptoms.

\section{Box 2: Indications for exercise testing in patients with no} symptoms of coronary artery disease ${ }^{10}$

Indication

- Patients with diabetes who are considering starting an exercise program*

- Patients with multiple risk factors for whom risk-reduction therapy may be guided by stress testing $\dagger$

- Men aged > 45 yr or women aged > 55 yr who:

- Plan to start vigorous exercise programst

- Are involved in occupations in which impairment might affect public safety (e.g., airline pilots, commercial truck drivers, bus drivers) $\dagger$

- Are at increased risk of coronary artery disease because of other diseases, such as peripheral vascular disease and chronic renal failuret

* Level of evidence lla: weight of evidence or opinion is in favour of usefulness or efficacy. tLevel of evidence llb: usefulness or efficacy is less well-established by evidence or opinion. symptoms and has a relatively high rate of falsepositive results. ${ }^{8}$ False-positive results can lead to increased downstream testing, overtreatment and unnecessary patient anxiety. A large cohort study found that $1.7 \%$ of patients who had an exercise test eventually (even after stress imaging) went on to coronary angiography, which carries a risk of complications, including a $0.1 \%$ risk of death. ${ }^{9}$ The lack of clear benefit along with the increased risk of potential harm led the American Heart Association and American College of Cardiology guideline to conclude that routine stress testing is not recommended for patients with no symptoms and a low or medium 10-year risk of CAD. ${ }^{10}$ There are some instances in which exercise testing may be considered for these patients (Box 2). ${ }^{10}$

Although stress imaging is highly sensitive and is a useful diagnostic test in patients with symptoms or high risk, limited evidence supports its use in patients with no symptoms and a low pretest probability of disease. Stress imaging and advanced noninvasive imaging add little value to risk assessments in this population and should not be done in this patient's case, given his risk profile (Box 1). ${ }^{6}$

\section{Case revisited}

Because of the patient's sex and age, in addition to his family history, he underwent screening for risk factors for CAD. The patient's BMI was 26 , his blood pressure was $130 / 80 \mathrm{~mm} \mathrm{Hg}$, his fasting blood glucose level was normal, his estimated glomerular filtration rate was 96 [normal 90-120] $\mathrm{mL} / \mathrm{min}$ per $1.73 \mathrm{~m}^{2}$, his total cholesterol level was $5.2[$ normal $<5.2] \mathrm{mmol} / \mathrm{L}$, his HDL cholesterol level was 1.5 [normal > 1.0] $\mathrm{mmol} / \mathrm{L}$ and his triglyceride level was 1.5 [normal $<1.7] \mathrm{mmol} / \mathrm{L}$. The patient's 10-year CAD risk using the Framingham risk score was 5.6\%, which places him in the low-risk category.

Given that the patient is considered to have low risk and has no symptoms, neither screening ECG nor an exercise stress test was performed. The patient was encouraged to maintain a healthy body weight and engage in moderate to vigorous exercise for 30-60 minutes most days of the week.

\section{References}

1. National Cholesterol Education Program (NCEP) Expert Panel on Detection, Evaluation, and Treatment of High Blood Cholesterol in Adults (Adult Treatment Panel III). Third Report of the National Cholesterol Education Program (NCEP) Expert Panel on Detection, Evaluation, and Treatment of High Blood Cholesterol in Adults (Adult Treatment Panel III) final report. Circulation 2002;106:3143-421.

2. Mancini GB, Gosselin G, Chow B, et al. Canadian Cardiovascular Society guidelines for the diagnosis and management of stable ischemic heart disease. Can J Cardiol 2014;30:837-49.

3. Anderson TJ, Gregoire J, Hegele RA, et al. 2012 update of the 
Canadian Cardiovascular Society guidelines for the diagnosis and treatment of dyslipidemia for the prevention of cardiovascular disease in the adult. Can J Cardiol 2013;29:151-67.

4. Sutherland SE, Gazes PC, Keil JE, et al. Electrocardiographic abnormalities and 30-year mortality among white and black men of the Charleston Heart Study. Circulation 1993;88:2685-92.

5. Pignone M, Fowler-Brown A, Pletcher M, et al. Screening for asymptomatic coronary artery disease. Rockville (MD): US Preventive Services Task Force Evidence Syntheses; 2003.

6. Canadian Cardiovascular Society. Five things physicians and patients should question. Available: www.choosingwiselycanada .org/recommendations/cardiology/ (accessed 2015 Apr. 13).

7. Aktas MK, Ozduran V, Pothier CE, et al. Global risk scores an exercise testing for predicting all-cause mortality in a preventive medicine program. JAMA 2004;292:1462-8.

8. Fowler-Brown A, Pignone M, Pletcher M, et al. Exercise tolerance testing to screen for coronary heart disease: a systematic review for the technical support for the US Preventive Services Task Force. Ann Intern Med 2004;140:W9-24.

9. Cournot M, Taraszkiewicz D, Galinier M, et al. Is exercise testing useful to improve the prediction of coronary events in asymptomatic subjects? Eur J Cardiovasc Prev Rehabil 2006; 13:37-44.

10. Gibbons RJ, Balady GJ, Bricker JT, et al. ACC/AHA 2002 guideline update for exercise testing: summary article: a report of the American College of Cardiology/American Heart Association Task Force on Practice Guidelines (Committee to Update the 1997 Exercise Testing Guidelines). Circulation 2002;106:1883-92.

Affiliations: Women's College Hospital Institute for Health Systems Solutions and Virtual Care (Bhatia, Pendrith); Peter Munk Cardiac Centre (Bhatia, Ross), Toronto General Hospital; Faculty of Medicine, University of Toronto (Bhatia, Ross), Toronto, Ont

Contributors: All of the authors participated in drafting and revising the manuscript, approved the version to be published and agreed to act as guarantors of the work.
Members of the Canadian Cardiovascular Society Choosing Wisely Canada Committee: Bill Ayach, Institute of Medicine, Cleveland Clinic, Cleveland, Ohio, and Division of Cardiology, University of Manitoba, Winnipeg, Man.; Sacha Bhatia, Women's College Hospital Institute for Health Systems Solutions and Virtual Care, Peter Munk Cardiac Centre, and Faculty of Medicine, University of Toronto, Toronto, Ont.; Ian Burwash and Ross Davies, Division of Cardiology, University of Ottawa Heart Institute, Ottawa, Ont.; Michelle Graham, Division of Cardiology, University of Alberta, and Mazankowski Alberta Heart Institute, Alberta Health Services, Edmonton, Alta.; Camille Hancock-Friesen, Faculty of Medicine, Dalhousie University, and Pediatric Cardiac Surgery, IWK Health Centre, Halifax, NS; David Marr, Faculty of Medicine, Dalhousie Medicine New Brunswick and Memorial University of Newfoundland, and Division of Cardiology, Saint John Regional Hospital, Saint John, NB; Blair O'Neill, Division of Cardiology, University of Alberta, Edmonton, Alta.; Normand Racine, Department of Medicine and Cardiology, Montréal Heart Institute, Montréal, Que.; Heather Ross, Peter Munk Cardiac Centre, and Faculty of Medicine, University of Toronto, Toronto, Ont.; and Chris Simpson, Division of Cardiology, Queen's University, Kingston, Ont.

CMAJ is collaborating with Choosing Wisely Canada (www.choosingwiselycanada.org), with support from Health Canada, to publish a series of articles describing how to apply the Choosing Wisely Canada recommendations in clinical practice. 